Proyecciones

Vol. $13 N^{\circ} 1$, pp. 25-34 July 1994

[iniversidad Católica del Norte

Antofagasta - Chile

\title{
SADDLE CONNECTIONS IN PARABOLIC DIFFERENTIAL EQUATIONS
}

\author{
C. MIGUEL BLAZQUEZ and ELIAS TUMA*
}

Universidad Técnica Federico Santa María, Valparaiso-Chile.

\begin{abstract}
We assume the existence of a saddle connection between two hyperbolic equilibrium points. Necessary and sufficient conditions are given for the existence of a connection for the perturbed equations. These connections are obtained from the zeros of a finite number of bifurcation functions.
\end{abstract}

"This research was partially supported by FONDECYT under grant 1931118 and Universidad Técnira Federico Santa María under grant 93.12.07 


\section{Introduction}

In the study of autonomous equations which present closed heteroclinic contours or homoclinic solutions, two approaches have become very interesting: the generation of periodic orbits when an autonomous perturbation is added to the equation [1], [3], [4], [12] and the existence of solutions which present chaotic behaviour, when considering the equation under suitable non autonomous periodical perturbations $[2],[5]$.

In order to determine if the connections are or are not maintained for the perturbed equations, it is necessary to study the conditions for the perturbations.

Hale and Lin [7] have already considered autonomous perturbations to retarded functional differential equations. In [1], [2] autonomous and periodic perturbations were considered to parabolic equations, when the unstable manifolds of the equilibrium points have the same finite dimension.

Here we study the existence of saddle connections under perturbations of evolution equations, where the unstable manifold of the equilibrium points have not necessarily the same dimension.

For the case of the existence of a heteroclinic contour, it can be deduced from (3.5) that the condition of the dimension equality of the unstable manifolds is convenient for the existence of isolated saddle connections in that contour.

A linear operator $A$ on a Banach space $X$ is said to be sectorial if $A$ is a closed, densely defined operator, such that, for some $\phi \in(0, \pi / 2)$ and some $\mu \geq 1$ and real $a$, the sector

$$
S_{a, \phi}=\{\lambda|\phi \leq| \arg (\lambda-a) \mid \leq \pi, \lambda \neq a\}
$$

is in the resolvent set of $A$, i.e. $S_{a, \phi} \cap \sigma(A)=\phi$, and

$$
\|(\lambda-A)^{-1}|| \leq \mu /|\lambda-a| \text { for all } \lambda \in S_{a, \phi} .
$$

Many elliptic boundary-value problems define sectorial operators as shown by Friednuan [6] and Pazy [11].

For any sectorial operator $A$, there is a real number $a$, such that, if $A_{1}=A+a l$, then $\operatorname{Re} \sigma\left(A_{1}\right)>0$. We can define fractional powers $A_{1}^{\alpha}$ of $A_{1}$. Consider the set $X^{\alpha} \subseteq X$ defined by the domain of $A_{1}^{\alpha}, X^{\alpha}=D\left(A_{1}^{\alpha}\right)$, with the graph norm $\|x\|_{\alpha}=\left\|A_{1}^{\alpha} x\right\|, x \in X^{\alpha}$. Different choices of a give equivalent norms on $X^{\alpha}[9$, Theorem 1.4.6]. These spaces $X^{\alpha}$ are Banach spaces in the norm \|\|$_{\alpha}$ for $\alpha \geq 0$. $X^{\alpha}$ is a dense subspace of $X^{\beta}$ with continuous inclusion for $\alpha \geq \beta$ [9, Theorem 1.4.8].

We assime that the equation

$$
\dot{x}+A x=g(x)
$$

where $A$ is a sectorial operator on a Banach space $X, g \in C^{k}\left(X^{\alpha}, X\right), k \geq 2$, has two hyperbolic equilibrium points $x_{1}$ and $x_{2}$, such that its linear approximation 
has a finite number of eigenvalues with negative real part and the rest of the spectrum has positive real part, i.e. $\operatorname{dim} W^{u}\left(x_{i}\right)=n_{i} ; i=1,2$. We also assume the existence of a solution of (1.1), which is a saddle connection $\Gamma=\{p(t) \mid t \in \mathbb{R}\}$ from $x_{1}$ to $x_{2}$, i.e.

$$
\lim _{t \rightarrow-\infty} p(t)=x_{1} . \quad \lim _{t \rightarrow+\infty} p(t)=x_{2}
$$

which approaches the equilibrium point tangent to eigenvectors corresponding to real and simple eigenvalues. Our aim is to determine the conditions under which, the perturbed equation of (1.1):

$$
\dot{x}+A x=f(x)
$$

where $f \in\left(^{k}\left(X^{\alpha}, X\right), k \geq 2\right.$, in a neighourhood of $g$ has a heteroclinic connection $\Gamma_{f}$ close to $\Gamma$ from an equilibrium point $x_{1 f}$ to an equilibrium point $x_{2 f}$, close to $x_{1}$ and $x_{2}$ respectively, and the $T$-periodical perturbed equation

$$
\dot{x}+A x=q(t, x)
$$

where $q \in C^{k}\left(\mathbb{R} \times X^{k}, X\right), k \geq 2$, in a neigbourhood of $g$ has a heteroclinic connection $\Gamma_{q}$ close to $\Gamma$, from a periodical solution $x_{1 q}(t)$ to a periodic solution $x_{2 q}(t)$ in neiglibourhoods of $x_{1}$ and $x_{2}$ respectively, which are given in (3.5) and (4.4) respectively. This last relation is valid in general for bounded perturbations.

\section{Preliminaries}

Suppose $A_{0}$ is a sectorial operator in $X, 0 \leq \alpha<1, t \rightarrow A(t)-A_{0}: \mathbb{R} \rightarrow L\left(X^{\alpha}, X\right)$ is locally Hölder continuous with exponent greater than $\alpha$ and let $T(t, s)$ be the solution operator for $\dot{x}+A(t) x=0$.

Definition 2.1 The equation $\dot{x}+A(t) x=0$ is said to have an exponential dichotomy on $J \subseteq \mathbb{R}$ with exponent $\beta>0$ and bound $M$ if there exist projections $P(t), Q(t)=I-P(t)$, such that

(a) $T(t, s) P(s)=P(t) T(t, s), \quad t \geq s$ in $J$.

(b) The restriction of $T(t, s)$ to the range of $Q(s)$, i.e. $\left.T(t, s)\right|_{R(Q(s))}, t \geq s$ is an isomorphism onto $R(Q(t))$ and we define the inverse map from $R(Q(t))$ to $R(Q(s))$ by $T(s, t)$.

(c) $\|T(t, s) P(s)\| \leq M e^{-\beta(t-s)}$ for $t \geq s$ in $J$.

(d) $\|T(t, s) Q(s)\| \leq M \epsilon^{3(t-s)}$ for $s \geq t$ in $J$.

\section{Rewarks}

1. We note that if $A(t)=A$ is a constant operator and, for some $\delta>0$, $\sigma(A)$ is disjoint from the strip $\{\lambda \mid-\delta \leq \operatorname{Re} \lambda \leq \delta\}$ then the equation has 
an exponential dichotomy on $\mathbb{R}$. This case holds for the linear variational equations

$$
\dot{x}+\left(A-g_{x}\left(x_{i}\right)\right) x=0 \quad i=1,2 .
$$

because $x_{i}$ are hyperbolic points.

2. If we consider the equation

$$
\dot{x}+\left(A-g_{x}(p(t)) x=0\right.
$$

where $\Gamma=\{\rho(t)\}$ is the saddle connection from $x_{1}$, to $x_{2}$, then by Roughness 'Theorem ([9, Theorem 7.6.11]) there exists a $T_{0} \in \mathbb{R}$, such that this equation has an exponential dichotomy on $\left(-\infty,-T_{0}\right]$ and on $\left[T_{0}, \infty\right)$. But for a general non-autonomous linear semigroup for which $R(Q(t))$ is finite dimensional, Lin [10] proved that the exponential dichotomy can be extended from $J=\left(-\infty, T_{0}\right]$ to $J=\left(-\infty, t_{0}\right], T_{0} \leq t_{0}$, under the assumption that $T\left(t_{0}, T_{0}\right) \phi \neq 0$ for any $\phi \neq 0 \phi \in R(Q(T))$. Also, the dichotomy can be extended from $J=\left[T_{0}, \infty\right)$ to $J=\left[t_{0}, \infty\right), t_{0} \leq T_{0}$, provided that $T^{*}\left(t_{0}, T_{0}\right) \psi \neq 0$ for any $\psi \neq 0, \psi \in R\left(Q^{*}\left(T_{0}\right)\right)$. these assumptions are satisfied in many application.

In what follows we suppose that the above assumptions are satisfied.

A result, for the existence of bounded solutions is given by

Theorem 2.2 Let us suppose that the equation

$$
\dot{x}+A(t) x=0
$$

has an exponential dichotomy on $(-\infty, \infty)$ with constant $M$ and exponent $\beta$, where $A(t)=A-B(t), A$ is a sectorial operator and $t \rightarrow B(t)$ is Hölder continuous.

If $f(t, x)$ is a continuous function such that $f(t, 0)=0$ and $\| f\left(t, x_{1}\right)-$ $f\left(t, x_{2}\right)\|\leq \gamma\| x_{1}-x_{2} \|_{\alpha}$ for $\left\|x_{1}\right\|_{\alpha},\left\|\left.x\right|_{2}\right\|_{\alpha} \leq \rho, t$ is real and $\gamma \eta M<1, b(t)$ is a continuous function such that

$$
\|b(t)\|=\sup |b(t)| \leq \frac{\rho(1-\gamma \eta M)}{\eta M},
$$

with

$$
\eta=\max \left\{\frac{2}{\beta}, \frac{1}{\beta}+\frac{\Gamma(1-\alpha)}{\beta-(1+\alpha)}\right\},
$$

where $\Gamma(t)$ is the gamma function.

Then the equation

$$
\dot{x}+A(t) x=b(t)+f(t, x)
$$

has a unique bounded solution $x(t)$ such that $\|x(t)\|_{\alpha} \leq \rho$.

Proof.

Let

$$
H_{\rho}=\left\{x: \mathbb{R} \rightarrow X^{\alpha} \mid x \text { is bounded, continuous and }\|x\|_{\alpha} \leq \rho\right\}
$$


and for $x \in H_{\rho}$. We define

$$
R(x)(t)=\int_{-\infty}^{t} T(t, s) P(s)\left(b(s)+f(s, x(s)) d s-\int_{t}^{\infty} T(t, s) Q(s)(b(s)+f(s, x(s)) d s\right.
$$

Then:

1. $R(x) \in H_{\rho}$, because $[\mathbf{9}$, Lemma 7.6.2]

$$
\begin{gathered}
\|R(x)(t)\|_{\alpha} \leq \int_{-\infty}^{t} M e^{-\beta(t-s)} \max \left\{1,(t-s)^{-\alpha}\right\}\|b(s)+f(s, x(s))\| d s \\
+\int_{t}^{\infty} M e^{-\beta(s-t)}\|b(s)+f(s, x(s))\| d s \leq \\
M\left(\frac{\rho(1-\gamma \eta M)}{\eta M}+\gamma \rho\right)\left[\int_{-\infty}^{t} e^{-\beta(t-s)} \max \left\{1,(t-s)^{-\alpha}\right\} d s+\int_{t}^{\infty} e^{-\beta(s-t)} d s\right] \leq \\
\frac{\rho}{\eta}\left(\int_{-\infty}^{t} e^{-\beta(t-s)} \max \left\{1,(t-s)^{-\alpha}\right\} d s+\int_{t}^{\infty} e^{-\beta(s-t)} d s\right) \leq \\
\frac{\rho}{\eta}\left(\int_{0}^{\infty} e^{-\beta u} \max \left\{1, u^{-\alpha}\right\} d u+\int_{0}^{\infty} e^{-\beta u} d u\right) \leq \rho
\end{gathered}
$$

2. $R$ is a contraction, because

$$
\begin{gathered}
\|R(x)(t)-R(y)(t)\| \leq \int_{-\infty}^{t} \max \left\{1,(t-s)^{-\alpha}\right\} M e^{-\beta(t-s)}\|f(s, x(s))-f(s, y(s))\| d s- \\
\int_{t}^{\infty} M e^{-\beta(t-s)}\|f(s, x(s))-f(s, y(s))\| d s \leq \\
M \gamma\|x(t)-y(t)\|_{\alpha}\left\{\int_{0}^{\infty} e^{-\beta u} \max \left\{1, u^{-\alpha}\right\}+\int_{0}^{\infty} e^{-\beta \mu} d u\right\} \leq M \eta \gamma\|x-y\|_{\alpha}
\end{gathered}
$$

As a consequence of this theorem, if the equation

$$
\dot{z}+A z=g(t, z)
$$

has a bounded solution $\xi(t)$ on $(-\infty, \infty)$, such that the variational equation

$$
\dot{z}+A z=g_{x}(t, \xi(t)) z
$$

has an exponential dichotomy on $(-\infty, \infty)$, then the perturbed equation:

$$
\dot{z}+A z=b(t, z)
$$

where $b$ is a bounded function in $t$, in a neigbourhood of $g$, has a unique bounded solution near $\xi(t)$.

However, if the equation (2.3) is autonomous and $\xi(t)$ is not an equilibrium point, then the Theorem 2.2 can not be applied, because the variational equation 
has now the nontrivial hounded solution $\dot{\xi}(t)$ and hence cannot have an exponential dichotomy on $(-\infty, \infty)$.

Findly we require an important lemma ([2]).

\section{Lemma 2.3}

If $A$ is a sectorial operator, $t \rightarrow A(t)-A: \mathbb{R} \rightarrow L\left(X^{\alpha}, X\right)$ is locally Hölder continuous, such that the equation

$$
\dot{x}+A(t) x=0
$$

has an exponential dichotomy on $\mathbb{R}^{+}$and $\mathbb{R}^{-}$with projections $P^{+}(t), Q^{+}(t)$ and $P^{-}(t), Q^{-}(t)$, respectively, with $R\left(Q^{+}(t)\right)$ and $R\left(Q^{-}(t)\right)$ of finite dimension. 'Then, for the linear operator

$$
L: C^{k+1}\left(\mathbb{R}, X^{\alpha}\right) \rightarrow C^{k}(R, X), \quad k \geq 0
$$

defined by

$$
(L x)(t)=\dot{x}(t)+A(t) x(t)
$$

we have:

(a) $L$ is Fredholm:

(b) $N(L)=\left\{g \in C^{k+1}\left(\mathbb{R}, X^{\alpha}\right): g(t)+A(t) g(t)=0, g(0) \in R\left(P^{+}(0)\right) \cap\right.$ $\left.R\left(Q^{-}(0)\right)\right\}$

(c) $R(L)=\left\{f \in C^{k}(\mathbb{R}, X): \int_{-\infty}^{\infty}\langle\psi(t), f(t)\rangle d t=0\right\}$,

for all $\psi \in C_{b}^{k+1}\left(\mathbb{R}, X^{\alpha *}\right)$ satisfying the adjoint equation

$$
\dot{x}-A^{*}(t) x=0
$$

and index $L=\operatorname{dim} R\left(Q^{-}(0)\right)-\operatorname{dim} R\left(Q^{+}(0)\right)$.

Where $N(L)$ and $R(L)$ are the kernel and the range of the linear operator $L$.

\section{Autonomous perturbation case}

Since the saddle points $x_{i}$ are hyperbolic then equation (2.1) has an exponential dichotomy. By Theorem 2.2 the perturbed equation has two hyperbolic, isolated saddle point, $x_{1 f}, x_{2}$ close to $x_{1}$ and $x_{2}$ respectively, which without lost of generality we consider that they coincide, i.e. $x_{1 f} \equiv x_{1}$ and $x_{2 f} \equiv x_{2}$.

By the remark 2, after definition 2.1 , we have that the variational equation $(2.2)$

$$
\dot{x}+\left[A-g_{x}(p(t))\right] x=0
$$

has an exponential dichotomy only on $\mathbb{R}^{-}$and $\mathbb{R}^{+}$.

We apply the results from the above section to obtain a bifurcation function for heteroclinic orbits of $(1.2)$, for $f$ near $g$. To this end, let $x(t)=p(t)+z(t)$ then

$$
\dot{z}+A(t) z=F(t, z)
$$


where

$$
A(t)=A-\frac{\partial g}{\partial x}(p(t)) \text { and } F(t, z)=f(p+z)-g(p)-\frac{\partial g}{\partial x}(p(t)) z
$$

Obtaining a heteroclinic orbit of the equation (1.4) is equivalent to obtain a small solution $z(t)$ of (3.1).

From the Roughness Theorem and the extension given by Lin [10], (see Remark 2), we have that the equation $\dot{z}+A(t) z=0$ has an exponential dichotomy on $\mathbb{R}^{+}$ and $\mathbb{R}^{-}$

We consider (3.1) as a perturbation of the equation

$$
(L z)(t)=0 \quad L=\frac{\partial}{\partial t}+A(\cdot) .
$$

and the operator $L$ as a continuous linear operator from $C^{k+1}\left(\mathbb{R}, X^{\alpha}\right)$ to $C^{k}(\mathbb{R}, X), k \geq 0$.

We construct the desired solution $z(t)$ of (3.1) by applying the Liapunov Schmidt method to obtain the bifurcation equations for bounded solutions of (3.1) (see Hale [8]).

Let $E_{2}$ a projection from $C^{k}(\mathbb{R}, X)$ to $R(L)$, and $E_{1}$ a projection from $C^{k+1}\left(\mathbb{R}, X^{\alpha}\right)$ to $N(L)$.

Then, the equation (3.1) is equivalent to the systems

$$
\begin{gathered}
\dot{z}+A(t) z=E_{2} F(t, z) \\
0=\left(i-E_{2}\right) F(t, z)
\end{gathered}
$$

Since $L$ is Fredholm, then it is known that the equation (3.2) can be replaced by

$$
\left(I-E_{1}\right) z=K E_{2} F(t, z)
$$

where $K: R\left(E_{2}\right) \rightarrow R\left(I-E_{1}\right)$, is the right hand side inverse of $L$.

We want to obtain bifurcation functions such that its zeros are in 1 - 1 correspondence with the heteroclinic connections of equation (1.2).

Let $\operatorname{dim} N(L)=d$, then we can consider $\left\{\dot{p}, y_{1}, y_{2}, \cdots, y_{d-1}\right\}$ as a basis for $N(L)$, so $z=\S+\sum_{i=1}^{d-1} k_{i} y_{i}$ where

$$
\widehat{z} \in R\left(I-E_{1}\right)=C^{k+1}\left(\mathbb{R}, X^{\alpha}\right) \backslash N(L)
$$

The equation (3.4) is equivalent to

$$
\hat{z}=K E_{2} F\left(t, \hat{z}+\sum k_{i} y_{i}\right)
$$

Iising the Implicit Function Theorem we obtain the existence of a solution $z^{*}(s)$, with $s=\left(k_{1}, \cdots, k_{d-1}\right)$. 
Let $\psi^{\prime} 1, \psi_{2}, \cdots, \zeta^{*}$ be a basis for the bounded solutions for the adjoint equation (2.4). From equations (3.3) and (3.4), we obtain:

$$
G_{j}(s, F) \equiv \int_{-\infty}^{\infty}\left\langle\psi_{j}, F\left(t, z^{*}+\sum_{1}^{d-1} k_{i} y_{i}\right)\right\rangle d t=0 \quad j=1,2, \cdots, d^{*}
$$

The functions $G_{j}, j=1 \cdots d^{*}$ are the bifurcation functions and the perturbed equations (1.2) has a heteroclinic connection in a neighbourhood of $\Gamma$ if and only if $i_{j}(s, F)=0, \forall j$ for some $\|s\|<s_{0}$. The desired heteroclinic solution is given by

$$
x(t)=p(t)+z^{*}(s)(t)+\sum_{i=1}^{d-1} k_{i} y_{i}(t)
$$

\section{Bounded perturbation case}

Let us suppose that $g \in C^{k}\left(X^{\alpha}, X\right), k \geq 2$, and the equation $\dot{x}+A x=g(x)$ has a nonconstant bounded solution $p(t)$ such that the variational equation $(2.2)$

$$
\dot{x}+A x=g_{x}(p(t)) x
$$

has an exponential dichotomy on $\mathbb{R}^{+}$and $\mathbb{R}^{-}$with projections $P^{+}(t)$ and $P^{-}(t)$ respectively.

So, according to Lemma 2.3 the operator $L$ given by

$$
L(x)(t)=\dot{x}(t)+\left\{A-g_{x}(p(t))\right\} x(t)
$$

is a Fredholm operator.

Let us suppose now that $q(t, x) \in C^{k}\left(R \times X^{\alpha} ; X\right), k \geq 2$, in a neigbourhood of $g$ and is bounded in $t$. We want to study the perturbed equation

$$
\dot{x}+A x=q(t, x) .
$$

We change variables in a neighbourhood of $p(t)$ by $x(t)=p(t+\alpha)+z(t+$ $\alpha), \quad \alpha \in \mathbb{R}$.

If $x(t)$ is a solution of (4.2) and $t$ is replaced by $t-\alpha$, then $z(t)$ satisfies

$$
\dot{z}+A(t) z=F(t, z, \alpha)
$$

where $A(t)=A-g_{x}(p(t))$ and

$$
F(t, z, \alpha)=q(t-\alpha, p+z)-g_{x}(p) z-g(p)
$$

i.e., $L(z)(t)=F(t, z, \alpha)$. Now we can apply the Liapunov-Schmidt method to obtain the bifurcation equations for bounded solutions of (4.3), then (4.3) is equivalent to

$$
\dot{z}+A(t) z=E_{2} F(t, z, \alpha), \quad 0=\left(I-E_{2}\right) F(t, z, \alpha) .
$$


Since the operator $L$ is Fredholm, there exists a bounded right inverse operator $K$ of $L$, so the first equation can be replaced this time by

$$
\left(I-E_{1}\right) z=K E_{2} F(t, z, \alpha)
$$

where $K^{*}: R\left(E_{2}\right) \rightarrow R\left(I-E_{1}\right)$.

If we consider again $\left\{\dot{p}, y_{1}, \cdots, y_{d-1}\right\}$ as a basis for $N(L)$, and $\left\{\psi_{1}, \psi_{2}, \cdots, \psi^{*}\right\}$ as a basis for the bounded solutions of the adjoint equation, the bifurcation system is given by

$$
C_{j}(k, \alpha, F)=\int_{-\infty}^{\infty}\left\langle\psi_{j}, F\left(z^{*}+\sum_{1}^{d-1} k_{i} y_{i}, \alpha\right)\right\rangle d t=0 \quad j=1,2, \cdots, d^{*}
$$

To prove the existence of periodic orbit $x_{1}(t)$ and $x_{2}(t)$ close to $x_{1}$ and $x_{2}$ respectively we have to consider that the saddle points $x_{i}(i=1,2)$ are hyperbolic, so the variational equations (2.1) have an exponencial dichotomy on $\mathbb{R}$. From the roughness theorem we obtain thet the equation $(4.2)$ has an exponential on both half sides $\mathbb{R}^{-}$and $\mathbb{R}^{+}$.

But, on the other hand, if we apply theorem 2.2 , we obtain that the perturbed equation (4.2) has a unique bounded solution $x_{i}(t)(i=1,2)$ in a neigbourhood of $x_{i}$. Furthermore, if we apply, as in theorem 2.2 , the operator $R$ on the set $H_{\rho}$ of all bounded continuous functions $x(t)$, such that $\|x(t)\|<\rho$, but with the extra condition that $x(t)$ is $T$-periodic, then, if $q$ is a $T$-periodic function we have that the fixed point $x_{i}(t)(i=1,2)$ is also $T$-periodic.

\section{References}

[1] C: M. Blázquez, E. Tuma Heteroclinic bifurcation in Banach spaces Lectures Notes in Mathematics 1331(1988), 12-38.

[2] (… M. Blázquez Transverse homoclinic orbit in periodically perturbed parabolic equations. Non Linear Analysis 10, No 11J (1986 ),1277-1291.

[3] C. M. Blázquez Bifurcation from a homoclinic orbit in parabolic differential equations Proc. Roy. Soc. Edin.103A (1986), 265-274.

[4] S. M. Chow, B. Deng Homoclinic and heteroclinic bifurcations in Banach spaces Preprint. Michigan State University

[5] S. M. Chow, J. K. Hale An example of bifurcation to homoclinic orbits of Dif. Eq.37 J.(1980).

[6] A. Friedman Hold, Rinehart and Winston 1969. Partial differential equation.

[7] J. K. Hale, X. B. Lin Heteroclinic orbit for retarded functional differential equation LCDS Report 84:39 Brown University 1984 
[8] J. K. Hale Introduction to dynamic bifurcation. Lecture Notes in Mathematjes. Springer Verlag, 1057(1984).

[9] D. Henry Gemetric theory of semilinear parabolic equations. Lecture Notes in Mathematics, Spriuger Verlag, 810 (1981).

[10] X. B. Lin Exponential dichotonies and homoclinic orbit in retarded Functional Differential Equations LCDS Report 8439 Brown IIniwersity. may 1984.

[1] A. Pazy Simjgroups of linear operators and applications to partial differential wutuations Appl. Math. Sc. 44, Springer Verlag (1983)

[12] L. P. Silnition 119 On the generation of a periodic motion from trajertorjes dombly asymptotic to an equilitrium state of saddle type. Math. USSR Shomik, Mat, Shornik Ton 7 rol 6(1968), 119.

Received: October 8, 1993

Departamento de Matemática

Eniversidad Tócnica Foderico Santa Maria

Casilla $110-V$. Valparaiso-Chile 